\title{
Conflicto intergrupal, validación e influencia minoritaria inmediata y diferida ${ }^{1,2}$
}

\author{
GABRIEL MUGNY y JUAN ANTONIO PÉREZ \\ Universidad de Ginebra
}

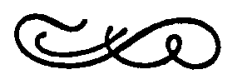

\section{Resumen}

Se presenta un experimento en el que se estudia la influencia minoritaria inmediata y diferida respecto a las opiniones frente al aborto y los anticonceptivos. Se ponen a prueba dos bipótesis: 1) La minoría obtendrá una influencia diferida cuando la conflictualidad que vehicula sea insertada en un contexto de oposiciones entre grupos. 2) Tal efecto deberä ser más notable cuando el blanco de influencia sea llevado a estimar, si los argumentos de la minoria merecen ser reflexionados (orientación sobre la validación), comparado a situaciones en las que tiene que expresar su grado de acuerdo con tales argumentos (resaltación de la comparación social). Los resultados confirman en gran medida esas bipótesis y son discutidos teniendo en cuenta las distancias ideológicas iniciales entre la fuente y el blanco de influencia, asi como la representación que éste ha elaborado de la minoría.

\section{Abstract}

An experience studies the immediate and delaved minoritv influence within a haradigm concerning the opinions on abortion and contraception, by testing a double hypothesis:

1) that a delayed influence is granted by a minority when the conflictual process concerned is included in a context of intergroup oppositions;

2) that this effect should be more obvious when the target have to decide wether the arguments brought up by the minority are worth to be taken into consideration (orientation towards valida. tion) rather than when they (the targets) bave to agree with the arguments (salience of the social comparison).

The results - that confirm fully these bypotheses-are looked at by taking into consideration the initial ideological distances between source and targets as well as the representation of the minority put forth by the targets.

Dirección del autor: Faculté de Psychologie et Sciences de l'Education, 1211 Gèneve, Suiza.

Agradecimientos:

(1) Este experimento ha sido realizado en el marco del Fondo Nacional Suizo de Investigación Científica, 1:367.0.86.

(2) Agradecemos a nuestros colegas del Laboratorio de Psicología Social de la Universidad Autónoma de Barcelona la ayuda que nos prestaron para poder llevar a cabo el experimento presentado aquí. 


\section{INTRODUCCION}

Está hoy ampliamente demostrado en una variedad de estudios (cf. Moscovici y Mugny, 1987) que la influencia minoritaria tiende a expresarse en forma de conversión: si bien la influencia que obtiene una mayoría a menudo se observa a nivel de las respuestas manifiestas (públicas, inmediatas, directas), por el contrario, la influencia de una minoría tiende a aparecer a nivel latente (Personnaz y Personnaz, 1987), privado (Martin, 1987), diferido (Moscovici, Mugny y Papastamou, 1981, Kaiser y Mugny, 1987), o sobre temas que no están directamente relacionados con el contenido expresamente defendido por la minoría (Aebischer, Hewstone y Henderson, 1984; Nemeth, 1986; Pérez, Mugny y Moscovici, 1986).

La noción clave a la que se ha recurrido para explicar estos fenómenos es la del conflicto. Se viene sosteniendo que la influencia minoritaria emerge de un conflicto; pero en realidad, en un primer momento la minoría sale desfavorecida de ese conflicto. Ello consituye la paradoja de la consistencia: por una parte la minoría debe mostrarse firme y no ceder en sus posiciones para obtener un impacto dado y, por otra parte, el efecto de ese mismo estilo de comportamiento consistente en un primer momento suele bloquear la influencia (Mugny, 1981). Así pues, dado el fuerte conflicto que induce una minoría consistente, de algún modo la misma minoría se crea las condiciones de su propio rechazo. Se trata claramente de una paradoja, puesto que la consistencia constituye al mismo tiempo la condición sine qua non (Moscovici, 1981) para que las posiciones minoritarias, que se caracterizan por una ruptura con las normas dominantes, sean percibidas como auténticamente alternativas y de este modo puedan llegar a constituir un nuevo punto de anclaje sociocognitivo para que el sujetoblanco de influencia reformule sus propias posiciones actitudinales.

Para poder conceptualizar esos efectos de resistencia, hemos sostenido (Mugny y Papastamou, 1982) que el estudio de la influencia social debe recurrir a los trabajos sobre las relaciones entre grupos (cf. Tajfel, 1982; Turner, 1987). En efecto, las minorías, dada su divergencia normativa, suelen ser percibidas como diferentes y a menudo son categorizadas como extragrupos o, en el mejor de los casos, quedan situadas en los confines del intragrupo (cf. Mugny y Pérez, 1985). En consecuencia, son propensas a ser discriminadas tanto a nivel evaluativo como comportamental, en concordancia con el funcionamiento sociocognitivo descrito por el modelo de la diferenciación categorial (Doise, 1979). De este modo, este modelo se muestra perfectamente adecuado para ayudarnos a comprender los aspectos manifiestos de la difícil influencia minoritaria. Como ya hemos sostenido en otra parte (Mugny, Rilliet y Papastamou, 1981), todo acercamiento manifiesto a una posición sostenida por un grupo, implica al mismo tiempo una identificación del blanco de influencia con dicho grupo. Esta identificación se traduce, concretamente, en una autoatribución de las características de la fuente resaltadás en el contexto social en el que se desarrolla la influencia. Se comprenderá fácilmente así porqué el blanco de influencia habitualmente se muestra reticente a asumir una identificación con una minoría cuando ésta ha sido categorizada como un extragrupo y/o cuando los atributos que le están asociados tienen una connotación negativa. En estos casos, toda identificación con la minoría amenazaría la identidad social del blanco de influencia y suscitaría un conflicto de identificación.

Dados estos fenómenos de discriminación, originados primordialmente en 
base a la división ideológica, la influencia minoritaria, en caso de producirse, lo más habitual es que adquiera una forma indirecta y diferida. Uno de los aspectos más interesantes de este fenómeno minoritario para la psicología social (cf. Mugny y Pérez, 1987) reside en que excluye toda explicación que recurra simplemente a un proceso de imitación o de modelo; exige sobre todo que se tenga que recurrir de entrada a un enfoque de los procesos de influencia social en términos socioconstructivistas: el blanco de influencia no adopta las posiciones minoritarias explícitas - dado el conflicto intergrupal en el que se inscriben-, y sin embargo tiende al mismo tiempo a desarrollar una actividad sociocognitiva compleja de la que resulta un cambio de actitud sobre temas que no constituyeron el objeto del intento de la influencia minoritaria (cf. Pérez et al., 1986). En este sentido, se ha mostrado que frente a una fuente minoritaria el blanco de influencia elabora respuestas que son más nuevas, más originales e incluso más correctas de las que elabora cuando se trata de una fuente mayoritaria (Nemeth, 1986). Estos efectos constructivistas emergen de una forma de pensamiento o funcionamiento sociocognitivo que se caracteriza por ser más intenso, más rico en cuanto a la información tratada y también más diversificado en cuanto a las dimensiones que se tienen en cuenta para organizar la información. Estas actividades cognitivas constituyen el llamado proceso de validación (Maass, 1987; Moscovici, 1980; Mugny y Pérez, 1986).

Dado que ese proceso de validación interviene primordialmente cuando se trata de posiciones que son propiamente innovadoras, se puede suponer que la discriminación de la minoría a nivel manifiesto va al mismo tiempo acompañada de una focalización de la atención del blanco de influencia sobre el objeto que constituye el litigio entre la mayoría y la minoría (cf. Personnaz y Guillon, 1985). Partiendo de este razonamiento, se puede suponer que el resentimiento de un tipo de conflicto interno (Moscovici y Personnaz, 1980) será más intenso cuando el blanco de influencia no haya podido resolver el conflicto a nivel mañifícsiuo. Las mayorías obtenárían menos infiuencia indirecta justamente porque ante ellas el conflicto sería resuelto a nivel manifiesto por medio de respuestas de complacencia; es decir, en este último caso, la resolución del conflicto se sitúa meramente a nivel de la comparación social. Frente a las minorías, por el contrario, la resolución del conflicto en cierta manera sería interiorizado, ya que tal conflicto perdura mientras los sujetos permanezcan en sus posiciones y la minoría afirme las suyas - por ejemplo, a través de la consistencia diacrónica, cf. Kaiser y Mugny, 1987. Es precisamente esta imposibilidad o dificultad psicosocial para resolver el conflicto a nivel manifiesto lo que, paradójicamente, desencadenaría esta actividad sociocognitiva de validación, actividad que constituye el proceso mediacional de los cambios indirectos o diferidos.

Ese razonamiento es indispensable para poder dar cuenta del conjunto de efectos paradójicos que se observan en la influencia minoritaria: el impacto indirecto o diferido de las minorías se manifiesta con más intensidad cuando la fuente es categorizada como una entidad distinta y distante, como un extragrupo (cf. Pérez y Mugny, 1987, con respecto a la influencia indirecta, y Mugny, Kaiser y Papastamou, 1983, respecto a la influencia diferida), o cuando se activan algunas resistencias a la innovación tales como la denegación (cf. Pérez et al., 1986). Partiendo de estos efectos podemos formular la siguiente hipótesis: el impacto minoritario estará facilitado por la conflictualización del campo social de la innovación, ya que de este modo las posiciones minoritarias, dada 
su oposición con las posiciones dominantes, aparecerán como auténticamente alternativas.

En el experimento que vamos a presentar a continuación trataremos de estudiar los efectos inmediatos y diferidos de un mensaje minoritario favorable al aborto. En unas condiciones intentaremos acentuar la conflictualización del campo social en el que se inscribe la minoría y las compararemos a otras en las que tal conflictualización habrá sido resaltada en menor grado. Como ya se habrá comprendido, nuestra predicción es que, como consecuencia de la división conflictual del campo de la innovación, la influencia de la minoría deberá disminuir a nivel inmediato, pero además, en esas mismas condiciones, se instaurará un proceso de influencia diferida más acentuado.

Trataremos de examinar aún otra hipótesis. Si es cierto que la validación se instaura en mayor grado cuando los sujetos están centrados sobre el objeto de litigio que cuando lo están sobre la comparación social - la fuente- (Moscovici y Personnaz, 1980), entonces se debería además obtener una acentuación de los efectos diferidos de la minoría cuando los sujetos tengan que indicar el grado en el que creen que el mensaje minoritario merece ser reflexionado (en última instancia uno se puede oponer a una idea y aceptar al mismo tiempo reflexionarla, lo que suele indicar que se le concede cierta validez) comparado a una situación en la que únicamente tengan que expresar su grado de adhesión con tal mensaje. En el primer caso se debería activar el proceso de validación y las minorias saldrían ganando, mientras que en el segundo se activaría el proceso de comparación social por el que las minorías saldrían perdiendo. Pasemos, pues, a ver los detalles metodológicos de este experimento y la concordancia de los resultados con estas ideas.

\section{METODO}

El experimento ha sido realizado con 116 alumnos y alumnas de 15 a 18 años de un instituto de enseñanza media situado en Barcelona. Con el fin de estudiar la influencia inmediata y diferida de una fuente minoritaria, el experimento se realizó en dos fases, con un intervalo de tres semanas entre una y otra.

Los sujetos comenzaron contestando a un breve pretest compuesto de cinco items referidos al aborto (en todos los cuestionarios se utilizaron escalas de 7 puntos: 1 = totalmente en desacuerdo, 7 = totalmente de acuerdo). Para el análisis de los resultados se ha dividido la población experimental en dos grupos iguales sobre la base de la mediana resultante de la suma de las respuestas a estos cinco items: los 58 sujetos que expresaron en este pretest las opiniones más favorables al aborto los llamaremos sujetos cercanos $(m=6,54)$, los 58 restantes sujetos alejados $(m=4,70)$. Como puede advertirse, de manera general los sujetos (recordemos que se trata de jóvenes) expresan una actitud bastante favorable al aborto, lo que no impedirá, como veremos, que reconozcan que la posición favorable al aborto tiene un carácter minoritario. De cualquier modo, lo más importante es que para los sujetos alejados se dará menor proximidad ideológica con el mensaje minoritario que para los sujetos cercanos.

Inmediatamente después del pretest se pasaba a la fase experimental propiamente dicha. Los sujetos debían leer un texto radicalmente favorable al aborto y explícitamente atribuido a un grupo minoritario. El texto estaba estructurado en función de dos dimensiones: en primer lugar resaltaba que es indispensable 
legalizar totalmente el aborto y que se elabore un estatuto jurídico que garantice el recurso voluntario al aborto. Por otra parte, el grupo minoritario reivindicaba en el texto que es indispensable que el aborto sea totalmente gratuito y que sus costos corran a cargo de la Seguridad Social (para más detalle puede verse: Pérez y Mugny, 1985). En el contexto hispano en general estos dos aspectos constituyen, desde el punto de vista normativo, una posición claramente minoritaria, confirmado también en este experimento, puesto que los sujetos juzgan la fuente como minoritaria $(m=5,15$, sobre una escala de siete puntos en la que 1 = mayoritario y $7=$ minoritario). No obstante, con el fin de conflictualizar la situación y a fin de asociar a la posición de la fuente un carácter normativamente minoritario, en todas las condiciones experimentales se introdujo una resistencia. Para ello se recurrió al procedimiento de la denegación que ya hemos utilizado en otras ocasiones (cf. Pérez et al., 1986). De este modo, antes de pasar a leer el texto minoritario, se pedía a los sujetos que indicasen de un total de cinco argumentos favorables al aborto, los cuatro que, en base a unos supuestos estudios que habíamos realizado con los jóvenes de ambos sexos, éstos los habrían juzgado como poco plausibles, no razonables y que no merecían ser tenidos en cuenta. Esto constituye un modo de recordar que la posición favorable al aborto constituye un punto de vista minoritario y que a éste le están asociados un conjunto de costos sociales (Mugny, Ibáñez, Elejabarrieta, Iñiguez y Pérez, 1986). De hecho, los sujetos indicaron que no eran razonables una media de 3,07 argumentos (sobre los 4 solicitados). Así pues, a pesar de que los sujetos tienden a estar a favor del aborto, también es cierto que no presentan grandes dificultades a la hora de indicar su carácter normativamente conflictivo.

Hasta aquí todos los sujetos han realizado las mismas tareas por este orden: el pretest, el procedimiento de denegación y la lectura del texto. Una vez terminada la lectura del texto se introdujo la manipulación experimental de las variables independientes. Para esto se recogieron en forma de proposición los

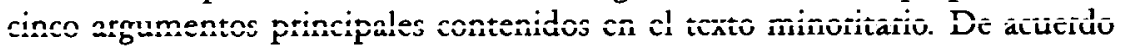
con la primera variable independiente, en la mitad de las condiciones los sujetos fueron llevados a centrarse en el grado de adhesión respecto a cada uno de los argumentos (condición adhesión); mientras que en la otra mitad eran centrados en el grado de reflexión que merecía cada uno de los argumentos (condición reflexión). La finalidad de esta variable independiente era centrar la atención de los sujetos ya sobre su acercamiento a la fuente (es decir, sobre la comparación social), o bien sobre el valor y la reflexión del contenido del mensaje (es decir, sobre la validación).

La segunda variable independiente se centró en manipular el contexto social en el que se debían expresar esos juicios. Para esto en todas las condiciones se evocaba el grupo de jóvenes; pero en la mitad de los casos se añadía una división ideológica dentro de esta categoría social resaltando la existencia de jóvenes conservadores y jóvenes progresistas (condición eintergrupo»); mientras que en la otra mitad de casos sólo se evocaba el grupo de jôvenes en general y no se introdujo la precedente división ideológica en el interior de esta categoría social (condición «intragrupo»). La finalidad de la manipulación de esta variable independiente era conflictualizar en mayor o menor grado el campo de la innovación intergrupal haciendo (o no) explícita la existencia de las divisiones ideológicas que en realidad atraviesan dicho campo.

El procedimiento concreto de estas manipulaciones fue el siguiente: la mitad 
de los sujetos (modalidad reflexión) debían indicar sobre escalas de tipo Likert el grado de reflexión que merecía acordarse a cada uno de los cinco argumentos que resumían el texto minoritario $(1=$ no merece ser reflexionado, $7=$ merece ser reflexionado). La otra midad de los sujetos (modalidad adhesión) debían indicar sobre un mismo tipo de escalas el grado de acuerdo o desacuerdo con cada uno de los cinco argumentos $(1=$ desacuerdo, $7=$ acuerdo $)$. En las condiciones dichas intergrupo los sujetos tenían que responder a tres cuestiones para cada uno de los cinco argumentos del texto: el grado de acuerdo (o reflexión) que atribuirían los jóvenes conservadores, el que atribuirían los jóvenes progresistas y por último el suyo personal. Por el contrario, en las condiciones dichas intragrupo en cada uno de los cinco argumentos sólo tenían que responder a dos cuestiones: el grado de acuerdo (o reflexión) que atribuirían los jóvenes en general y el suyo personal. Para analizar estas respuestas calcularemos la media de las respuestas de los cinco argumentos.

Después de estas manipulaciones se realizaron diversas medidas dependientes: en primer lugar los sujetos debían describir la fuente a través de una serie de escalas bipolares (que describiremos más adelante en su momento oportuno). Por último, se pasó a realizar la medida de la influencia de la fuente. Para esto los sujetos debían expresar su grado de acuerdo o de desacuerdo (escalas en 7 puntos: 7 = totalmente de acuerdo) con 25 items (18 referidos explícitamente a la legalización y gratuidad del aborto y los 7 restantes a la contracepción, cf. Pérez y Mugny, 1985).

El experimentador concluía esta fase experimental anunciando que volvería algunos días más tarde para continuar con algunos aspectos más del estudio; lo que efectivamente sucedía tres semanas después de esta primera fase. Durante esta segunda fase los sujetos tuvieron que responder, por supuesto $\sin$ volver a leer el texto minoritario y sin introducir ninguna manipulación experimental, al cuestionario de descripción de la fuente y a los 25 items relacionados con las opiniones frente al aborto y los anticonceptivos. Para obtener el índice de la influencia diferida hemos restado las respuestas del postest de esta segunda fase con las de la primera. Un signo positivo indicará un cambio diferido (conocido en la literatura del cambio de actitudes por el nombre de sleeper effect) hacia la posición minoritaria.

Antes de pasar a ver los resultados, señalemos que el análisis de varianza sobre los datos ha sido realizado siguiendo un plan factorial de $2 \times 2 \times 2$. Es decir, se compararán los efectos de las variables experimentales centración sobre la adhesión versus la reflexión $\times$ contexto intergrupo versus intragrupo $\times$ posición ideológica inicial de los sujetos alejados versus cercanos.

\section{RESULTADOS}

\section{a) Representación del contexto de la innovación}

Los sujetos de las condiciones intragrupo atribuyen a los jóvenes en general un juicio medio $(m=5,77)$ que se sitúa por encima del punto medio de la escala, lo que significa que nuestros sujetos se representan la posición de la juventud como ampliamente favorable al aborto. Independientemente de que este juicio haya sido expresado por sujetos alejados o por sujetos cercanos, e independientemente también de que se trate del grado de adhesión o bien de refle- 
xión. las respuestas que los sujetos piensan que darán los jóvenes en general a los argumentos sostenidos por el grupo minoritario no varían. Estos juicios se aproximan bastante a los que asignan los sujetos de las condiciones intergrupo a los jóvenes progresistas $(m=6.10)$, y ambos se contrastan fuertemente con los asignados a los jóvenes conservadores $(m=2,78)$.

Estos resultados ponen de manifiesto dos aspectos. En primer lugar, lo más esencial para nuestra intención aquí es que la diferenciación ideológica dentro de la categoría de los jóvenes ha sido claramente acentuada en las condiciones en las que los sujetos tenían que juzgar por separado las actitudes probables de los jóvenes progresistas y de los jóvenes conservadores. En segundo lugar, cuando no se introdujo ninguna diferenciación ideológica intracategorial entre los jóvenes, es decir, cuando se trata de siruar a los jóvenes en general, se expresa una representación más bien progresista de éstos. Por lo tanto, en todas las condiciones experimentales los sujetos disponen de un grupo de referencia similar: los jóvenes (que globalmente son percibidos como progresistas). Ahora bien, en la mitad de las condiciones este carácter progresista de los jóvenes, constrastará con que también existen jóvenes conservadores; la presencia simbólica de esta subcategoría contribuye en estas condiciones a conflictualizar el campo social.

Estos efectos constatados a nivel de la inducción experimental misma, nos sitúan en condiciones ideales para comparar los efectos de la categoría de referencia de los jóvenes en función de si se ha acentuado o no el contexto de oposiciones ideológicas. De acuerdo con nuestras conjeturas, esta conflictualización del campo de la innovación es la que debería inducir las dinámicas de una diferenciación con respecto a la minoría a nivel manifiesto, pero también de una conversión a nivel diferido.

\section{b) Posición del sujeto dentro del contexto de la innovación}

El examen de las respuestas a los cinco argumentos sostenidos por la minoría expresadas por los sujetes después de haber imputado unos u otros juicios a los jóvenes, pone de manifiesto que ya a este nivel aparecen algunas dinámicas de diferenciación personal. El primer efecto del que tenemos que partir es que globalmente el grupo de sujetos cercanos expresan opiniones más favorables $(m=6,01)$ hacia los argumentos del texto minoritario que el grupo de sujetos alejados $(\mathrm{m}=4,81 ; \mathrm{F} 1 / 108=22,205, \mathrm{p}<.001$; todos los niveles de significación estadística son bilaterales, el caso contrario será precisado), lo que no conlleva nada de extraño. El único interés de este resultado es que nos permite pasar a precisar en qué zona del campo social se sitúan realmente los sujetos a nivel personal.

De este modo, merece señalarse que los juicios personales de los sujetos cercanos $(m=6,01)$, se aproximan notablemente a la actitud atribuida por este grupo de sujetos a los jóvenes en general $(m=5,74)$, así como a la que han atribuido a los jóvenes progresistas $(m=6,46)$; y se oponen claramente a la que han asignado a los jóvenes conservadores $(m=2,82)$. Se puede concluir así que el grupo de sujetos cercanos en realidad se identifica con la franja más progresista de los jóvenes y que de este modo podría estar facilitada en mayor grado la identificación con la fuente minoritaria.

Las cosas suceden de otro modo en el grupo de los sujetos alejados. Los juicios personales de estos $(\mathrm{m}=4,81)$ se sitúan entre los que han atribuido a los jóvenes en general $(m=5,81)$ o a los jóvenes progresistas $(m=5,76)$, y los que 
imputaron a los jóvenes conservadores $(m=2,75)$. Para el grupo de sujetos alejados la resaltación de la división ideolólogica dentro del grupo de los jóvenes (en jóvenes progresistas y conservadores) debería ser una fuente de conflicto más intenso, puesto que se sentirían en mayor grado atraídos en dos direcciones opuestas, ambas plausibles para ellos.

Pero además, la expresión de los juicios personales por el grupo de sujetos cercanos y alejados varía en función de la naturaleza del contexto categorial manipulado (intergrupo versus intragrupo). En efecto, la interacción entre estas dos variables $(\mathrm{F} 1 / 108=4,593, \mathrm{p}<.04)$ indica que las dinámicas se invierten en función de si la posicion ideológica inicial de los sujetos está o no próxima de la dimensión progresista vehiculada por los jóvenes. Así, el grupo de sujetos alejados expresa una actitud personal más favorable (adhesión y reflexión confundidas) hacia los argumentos de la minoría cuando sólo se resalta la categoría de pertenencia global (los jóvenes en general, $m=5,12$ ) que cuando la categoría de referencia está dividida ideológicamente (contexto intergrupo, $\mathrm{m}=4,43$; $\mathrm{t} / 108=1,689, \mathrm{p}<.05$, hipótesis unilateral (1)). Más aún, dentro de este contexto conflictivo de oposición ideológica, este grupo de sujetos alejados muestra sobre todo una reticencia a adherirse a los argumentos de la minoría $(\mathrm{m}=4,17$; comparada a las tres condiciones restantes: $\mathrm{t} / 108=2,111, \mathrm{p}<.04$ ), mientras que el hecho de reconocer que los argumentos de la minoría son dignos de ser reflexionados está menos afectado $(m=4,84)$. En otros términos, se encontraría aquí una ligera confirmación de la hipótesis según la cual en un contexto conflictivo los sujetos ponen más reticencias a expresar su adhesión con los argumentos de la minoría y que, por el contrario, dado ese contexto conflictivo, «prefieren» pasar a otorgarles algún valor de reflexión, que de alguna manera valida la posición minoritaria.

Por contraste, el grupo de sujetos cercanos tiende a expresar una actitud más favorable frente a los argumentos minoritarios en el contexto intergrupal $(\mathrm{m}=6,27)$ que en el contexto intragrupal $(\mathrm{m}=5,82)$. Es decir, polarizan más sus juicios cuandos se resaltan las oposiciones ideológicas que dividen (¿amenazan?) su propio grupo de pertenencia; no obstante, no haremos hincapié en este resultado puesto que no es estadísticamente significativo $(t / 108=1,301$, $\mathrm{p}>.10)$.

No carece de interés realizar una interpretación de esta interacción partiendo de otro punto de vista: cuando se hace referencia a los jóvenes en general, la diferencia entre el grupo de sujetos alejados $(m=5,12)$ y el de los cercanos $(\mathrm{m}=5,82 ; \mathrm{t} / 108=1.972, \mathrm{p}<.001)$. Esta diferenciación induciría un efecto de polarización de los juicios de los sujetos cercanos $(m=6,27)$ y un efecto de «despolarización» en los sujetos alejados $(m=4,43)$. La conflictividad del campo social induciría en última instancia un efecto de bipolarización de los juicios personales, como ya la había señalado, concretamente, Paicheler (1977).

\section{c) Influencia inmediata}

Veamos ahora los resultados de la influencia propiamente dicha. En primer lugar es necesario señalar que un análisis por separado de las respuestas de los 18 ítems relacionados con el tema del aborto y los 7 referidos a los anticonceptivos, arrojan aproximadamente el mismo tipo de efecto (la correlación entre los ítems referidos al aborto y los referidos a los anticonceptivos es de +0.60 ). Así pues, a fin de simplificar la exposición de estos resultados, hemos calculado un 
sólo índice que engloba los 25 ítems del cuestionario de actitud contestado al final de la primera fase del experimento. Las medias correspondientes a las distintas condiciones experimentales figuran en la tabla $\mathrm{I}$.

TABLA 1

Influencia inmediata. Cuanto mayor es la puntuación, mayor es la influencia Entre paréntesis figura el nümero de sujetos.

\begin{tabular}{|c|c|c|c|c|c|}
\hline \multirow{3}{*}{ contexto } & $\begin{array}{c}\text { sujetos: } \\
\text { centración: }\end{array}$ & \multicolumn{2}{|c|}{$\begin{array}{c}\text { ALEJADOS } \\
\text { adhesión reflexión }\end{array}$} & \multicolumn{2}{c|}{$\begin{array}{c}\text { CERCANOS } \\
\text { adhesión reflexión }\end{array}$} \\
\hline \multirow{3}{*}{} & intragrupo & $\begin{array}{c}5,16 \\
(14)\end{array}$ & $\begin{array}{c}5,04 \\
(18)\end{array}$ & $\begin{array}{c}5,79 \\
(17)\end{array}$ & $\begin{array}{c}5,42 \\
(16)\end{array}$ \\
\cline { 2 - 6 } & intergrupo & $\begin{array}{c}4,57 \\
(16)\end{array}$ & $\begin{array}{c}4,57 \\
(10)\end{array}$ & $\begin{array}{l}5,68 \\
(12)\end{array}$ & $\begin{array}{c}6,21 \\
(13)\end{array}$ \\
\hline
\end{tabular}

El primer efecto que arroja el análisis de varianza es que sigue existiendo una diferencia clara entre los sujetos alejados $(m=4,86)$ y los cercanos $(m=5,76$; $\mathrm{F} 1 / 108=30,689, \mathrm{p}<.001)$. El segundo tipo de efecto se encuentra en la interacción entre el contexto categorial y la distancia ideológica inicial de los sujetos $(\mathrm{F} 1 / 108=6,801, \mathrm{p}<.01)$. De modo coherente con lo visto respecto a los juicios personales con respecto a los argumentos minoritarios y de acuerdo también con nuestras predicciones, el grupo de sujetos alejados se muestra menos favorable a las posiciones minoritarias en el contexto intergrupal $(m=4,57)$ que en el contexto intragrupal $(\mathrm{m}=5,09 ; \mathrm{t} / 108=2,260, \mathrm{p}<.02$, hipótesis unilateral). Así pues, en este grupo de sujetos alejados de las posiciones minoritarias, cuanto más se conflictualiza el contexto de la innovación, más se disminuye la influencia inmediata de la minoría: al resaltarles la existencia de juicios opuestos, en medio de los cuales se encuentran situados ellos mismos, expresan un acuerdo menos favorable con las posiciones próximas a las sostenidas por la minoría.

El ğíujo de sujeros cercanos tiende a expresar actitudes un poco más favorables con la dirección de las posiciones de la minoría en el contexto intergrupal $(\mathrm{m}=5,96)$ que en el contexto intragrupal $(\mathrm{m}=5,61)$, pero este efecto continúa sin ser significativo $(t / 108=1,476, p>.10)$.

Un análisis más detallado de los resultados de los sujetos cercanos pone de manifiesto que de hecho sólo una condición se ha mostrado eficaz en la influencia arrojada, comparada con las tres restantes $(\mathrm{t} / 108=2,107, \mathrm{p}<.04)$ : es la condición en la que se resaltó el contexto intergrupal y que además la atención de los sujetos fue centrada sobre la reflexión del discurso minoritario. Este efecto es coherente con nuestras hipótesis, pero ha aparecido antes de lo previsto, desde la primera fase, a nivel de la influencia inmediata. ¿Por qué? Se podría apuntar que para estos sujetos las resistencias han sido menores, dado que ya compartían ampliamente las opiniones de la fuente minoritaria. En cualquier caso, esta influencia ha aparecido en la condición de fuerte resaltación del contexto de oposición intergrupal y cuando tuvieron que pronunciarse sobre la «validez» de los argumentos minoritarios.

\section{d) Influencia diferida}

¿Qué sigue aconteciendo con esas dinámicas de influencia tres semanas más tarde? Los cambios de opiniones que ocurren entre el primer y el segundo pos- 
test (véase la tabla II) muestran que las dinámicas de la conversión han echado efectivamente sus raíces. Comparemos en primer lugar lo acontecido dentro de una misma condición entre el primer y el segundo postest, es decir, los cambios de opiniones en términos absolutos. Los efectos no son muy numerosos, pero sí suficientes para apuntar que nuestras hipótesis reciben alguna confirmación. Así, se observa que en las condiciones intergrupo, al calcular la diferencia entre el segundo y el primer postest, se ha producido un cambio positivo significativo $(\mathrm{m}=+0,16 ; \mathrm{t} / 50=1,85, \mathrm{p}<.036$ hipótesis unilateral $)$, cambio que no se constata en las condiciones intragrupo $(\mathrm{m}=+0,03 ; \mathrm{t} / 64=0,45$, n.s.). En la misma línea, la centración sobre la reflexión de los argumentos también tiende a producir un cambio diferido $(\mathrm{m}=+0,13 ; \mathrm{t} / 56=1,59, \mathrm{p}<.06$, hipótesis unilateral), cambio que no induce la centración en la adbesión $(m=+0,05$; $\mathrm{t} / 58=0,64$, n.s.).

Comparemos ahora más directamente las condiciones entre ellas para analizar los cambios de opiniones relativos. En primer lugar debe advertirse que a este nivel no se encuentra una diferencia global entre los sujetos alejados y los sujetos cercanos. Pero si se vuelve a encontrar una interacción entre el contexto categorial y la distancia ideológica inicial de los sujetos $(\mathrm{F} 1 / 108=4,508$, $\mathrm{p}<.04)$. En el grupo de los sujetos cercanos se encuentran pocas diferencias $(\mathrm{t} / 108=0,563$, n.s.) en función del contexto intergrupal $(\mathrm{m}=+0,04) 0$ intragrupal $(\mathrm{m}=+0,13)$, como si para este grupo de sujetos todo hubiese concluido ya en el primer postest.

\section{TABLA II}

Influencia diferida. Un signo positivo indica un cambio diferido

\begin{tabular}{|c|c|c|c|c|c|}
\hline \multirow{3}{*}{ contexto } & $\begin{array}{c}\text { sujetos: } \\
\text { centración: }\end{array}$ & \multicolumn{2}{|c|}{$\begin{array}{c}\text { ALEJADOS } \\
\text { adhesión reflexión }\end{array}$} & \multicolumn{2}{|c|}{$\begin{array}{c}\text { CERCANOS } \\
\text { adhesión reflexión }\end{array}$} \\
\cline { 2 - 6 } & intragrupo & $-0,16$ & 0,00 & $+0,07$ & $+0,19$ \\
\cline { 2 - 6 } & intergrupo & $+0,15$ & $+0,43$ & $+0,10$ & $-0,01$ \\
\hline
\end{tabular}

Por el contrario, en los sujetos alejados aparece una diferencia notable $(\mathrm{t} / 108=2,465, \mathrm{p}<.02)$. Estos sujetos dan muestras de un cambio diferido en la dirección de la posición minoritaria cuando se conflictualizó el campo social resaltando el contexto intergrupal $(\mathrm{m}=+0,26)$, y tal cambio no aparece en el contexto intragrupal menos conflictivo $(m=-0,07)$. Así pues, se encuentra un efecto de conversión en los sujetos más reacios a la alternativa minoritaria. Por último, merece subrayarse que dicho efecto es particularmente evidente en la condición en la que se acentuó el conflicto intergrupal y que además los sujetos fueron centrados sobre la «validación» del discurso minoritario («merece reflexión»: $m=+0.43$ ); esta condición difiere significativamente de las tres restantes en el sentido esperado $(t / 108=2,207, \mathrm{p}<.02$, hipótesis unilateral).

e) Análisis complementario: la representación de la minoría.

Se ha realizado un análisis factorial (con rotación varimax) sobre los 30 ítems (media de las respuestas inmediatas y diferidas) puestos a disposición de los 
sujetos para describir la fuente minoritaria. Del total de los ocho factores resultantes, sólo retendremos los tres que arrojan alguna diferencia entre las condiciones experimentales, y que por lo tanto pueden aportarnos alguna información sobre las dinámicas por las que han atravesado los efectos de influencia vistos. Uno de estos factores se refiere a la tolerancia de la minoría e incluye los ítems siguientes: tolerante, flexible, transigente y democrática. Otro hace referencia al estatus minoritario de la fuente, puesto que reagrupa los siguientes ítems: «no representa a nadie y marginal. El tercer factor califica el carácter crítico y atrevido de la intervención minoritaria. Las puntuaciones factoriales correspondientes a estos tres factores se encuentran en la tabla III.

\section{TABLA III}

Puntuaciones factoriales referentes a la imagen de la minoria (cuanto mayor es la puntuación más se aplica la "etiquetas a la minoría).

\begin{tabular}{|c|c|c|c|c|c|}
\hline \multirow[b]{2}{*}{ condiciones: } & \multirow{2}{*}{$\begin{array}{l}\text { sujetos: } \\
\text { centración: } \\
\text { contexto: }\end{array}$} & \multicolumn{2}{|c|}{ ALEJADOS } & \multicolumn{2}{|c|}{ CERCANOS } \\
\hline & & $\begin{array}{l}\text { adhesión } \\
\text { intra inter }\end{array}$ & $\begin{array}{l}\text { reflexión } \\
\text { intra inter }\end{array}$ & $\begin{array}{l}\text { adhesión } \\
\text { intra inter }\end{array}$ & $\begin{array}{l}\text { reflexión } \\
\text { intra inter }\end{array}$ \\
\hline & tolerancia: & $-0,09+0,03$ & $-0,20+0,19$ & $-0,23+0,44$ & $-0,34+0,51$ \\
\hline & minoritaria: & $+0,01+0,02$ & $+0,08+0,36$ & $+0,06-0,49$ & $-0,14+0,13$ \\
\hline & crítica: & $-0,25+0,11$ & $-0,36+0,62$ & $+0,16-0,38$ & $+0,01+0,28$ \\
\hline
\end{tabular}

Respecto a la dimensión de la tolerancia se encuentra el siguiente efecto global $(\mathrm{F} 1 / 108=9,250, \mathrm{p}<.003)$ : en el contexto intercategorial se reconoce la minoría como más tolerante $(\mathrm{m}=+0,22)$ que en el contexto intracategorial $(m=-0,28)$. En otros términos, la división del campo social hace resaltar la tolerancia que, por otra parte, ya sabemos que constituye el principio organizador de la posición minoritaria (Pérez y Mugny, 1986).

La cuestión central ahora es determinar por qụé las dinámicas de influencia más marcadas (a nivel inmediato) en los sujetos cercanos y a nivel diferido en los sujetos alejados han aparecido en el contexto intergrupal cuando los sujetos fueron llevados a pronunciarse sobre la reflexión que merecía otorgarse a los argumentos minoritarios. Dos interacciones entre el contexto categorial y la orientación de la atención de los sujetos tienden a ser significativas (en el factor minoritario: $F 1 / 108=2,850$, p $<.10 ;$ y en el factor cítico: $F 1 / 108=3,779$, $\mathrm{p}<.06)$ y nos aportan algún elemento para responder a tal pregunta. En primer lugar, aparece que, en esa condición que retiene nuestra atención, la minoría tiende a ser percibida como más minoritaria $(m=+0,23$; con respecto a las tres condiciones restantes: $(t / 108=1,603, p<.12)$. Pero sobre todo es la condición en la que se percibe la minoría como más crítica y atrevida $(\mathrm{m}=+0,43 ; \mathrm{t} / 108=2,473, \mathrm{p}<.02)$.

Este factor, referido al carácter crítico de la minoría, también arroja una interacción significativa entre la categorización del campo y la distancia ideológica $(\mathrm{F} 1 / 108=4,628, \mathrm{p}<.03)$ : para los sujetos alejados el contexto intergrupal hace resaltar más el carácter crítico de la minoría $(m=+0,30)$ que el contexto intracategorial $(\mathrm{m}=-0.30 ; \mathrm{t} / 108=2,538, \mathrm{p}<.02)$; mientras que para los sujetos cercanos tal diferencia no es de ningún modo significativa ( $t / 108=0,540$, n.s.). Estos resultados indicarían porqué fue sobre todo en los sujetos alejados en los que los efectos del contexto intergrupal sobre la influenciä observada fueron más significativos. Así pues, la difícil influencia inmediata, así como el efecto 
de conversión constatado en los sujetos alejados, encontraría claramente sus orígenes en la conflictualización del campo social de la innovación en base a la explicitación de las divergencias ideológicas que dividen a los jóvenes.

Dicho brevemente, el contexto de la división ideológica ha favorecido la tesaltación del principio organizador, del núcleo normativo, de las posiciones minoritarias: la tolerancia. A este efecto hay que añadir la búsqueda del valor de reflexión que, en un campo de división ideológico, hace resaltar de modo particular la percepción de la fuente como minoritaria y, sobre todo, como crítica. Así pues, la condición clave de este experimento es aquella en la que el principio organizador (la tolerancia) ha emergido en un contexto más conflictual, intergrupal, marcado de divisiones ideológicas explícitas, contexto en el que la minoría figura como una alternativa auténtica.

\section{CONCLUSIONES}

La convergencia de esta variedad de resultados viene a demostrar que las dinámicas específicas de la influencia minoritaria se desarrollan en un campo de tensiones entre posiciones ideológicas contradictorias. Así, en este experimento, caracterizado por la relativa proximidad ideológica de los sujetos con respecto a la fuente, incluso si ésta es reconocida como minoritaria en un contexto más amplio, el impacto más claro de la minoría aparece cuando el blanco de influencia es llevado a situarse personalmente frente a los jóvenes progresistas y los jóvenes conservadores (contexto intergrupal) en comparación con los jóvenes en general (contexto intragrupal). No obstante, las dinámicas que aparecen adoptan ritmos distintos en función de si los sujetos se sitúan dentro de las posiciones progresistas o si, a pesar de todo, se diferencian de éstas.

Los sujetos más cercanos de la minoría muestran un juicio personal muy próximo del que atribuyen a los jóvenes en general o a los jóvenes progresistas, y se alejan de las posiciones opuestas de los jóvenes conservadores. En el postest inmediato, la división ideológica explícita entre los jóvenes de «izquierdas» y los jóvenes de «derechas», produce un efecto de polarización de sus actitudes frente al aborto, y sobre todo cuando no tuvieron que expresar su grado de adhesión con las tesis minoritarias, sino indicar en qué medida el discurso minoritario merecía ser reflexionado. El contexto intergrupal hizo resaltar la tolerancia de la fuente (particularmente manifiesta ante la presencia de los jóvenes conservadores como punto de comparación) y la centración en la reflexión de los argumentos minoritarios ha añadido el carácter minoritario y crítico de la fuente, es decir, la especificidad de ésta en el campo de las posiciones ideológicas. La interpelación de los sujetos cercanos en tales condiciones hace aparecer inmediatamente el efecto, muy probablemente debido a que estos sujetos pueden identificarse más fácilmente con la fuente minoritaria. En este caso concreto, como en algunos otros estudios (cf. Mugny y Pérez, 1985: Mugny, Pérez, Raiser y Papastamou, 1984), es necesario tener en cuenta que el conflicto induce un mayor cambio de opiniones cuando el sujeto siente una pertenencia categorial o ideológica común con la minoría, es decir, cuando ambos reconocen que ya forman parte de un intragrupo.

Las cosas son bastante diferentes en el caso de los sujetos alejados, que, como se ha podido ver, se encuentran presionados entre las posiciones de los jóvenes conservadores y las de los jóvenes progresistas. También estos sujetos reconocen en mayor grado la tolerancia y la actitud crítica y atrevida que subyace a la alter- 
nativa minoritaria cuando las divisiones ideológicas implican una especie de costo social de la identificación en caso de aprobar abiertamente a la minoría. La consecuencia fue que esos sujetos se acercan menos a la posición minoritaria que cuando el filtro lo constituyó el contexto intragrupo. La conflictividad del campo social pone barreras a la influencia minoritaria a nivel inmediato, sobre todo cuando se ha resaltado el contexto intergrupal de la innovación.

Pero ese conflicto no impide que las ideas minoritarias abran su camino con el paso del tiempo. Son esos mismos sujetos los que mostrarán un mayor cambio diferido. Y éste ha sido más notable cuando no tuvieron que centrarse en el grado inmediato de adhesión con el texto minoritario, sino «simplemente» expresar si merecía o no llevarse a cabo una reflexión sobre sus argumentos, lo que aparentemente terminaron haciendo. Es así como han percibido en mayor grado el carácter alternativo de la minoría, su carácter crítico y tolerante. En un primer momento resistieron a su influencia, pero posteriormente se «atraparon» en un proceso de conversión.

Así pues, el impacto de las minorías parece producirse con mayor profundidad en un contexto conflictualizado por las divisiones del campo social de la innovación y cuando se induce explícitamente una validación de las posiciones minoritarias. No obstante, la resolución del conflicto para unos (los sujetos cercanos) puede realizarse a nivel inmediato, en forma de polarización de las posiciones, mientras que para otros (los más alejados) hay que esperar a la solución diferida.

La innovación inherente a la revelación de una posición desviada sostenida por una entidad minoritaria activa (Moscovici, 1981), induce, casi podríamos decir fatalmente, una conflictualización del contexto social. Es a través de la reconstrucción mental de este conflicto, de su representación en el blanco de influencia, de la que se desprende el impacto minoritario, impacto que se caracteriza por un posible rechazo o evitación a un nivel u otro (directo, público, inmediato) pero que se acompaña paradóịcamente al mismo tiempo de una u otra forma de cambio (indirecto, privado, diferido), que seguramente tiene algo de inconsciente (Moscovici y Mugny, 1987). Esta forma indirecta sería la más propensa a que se establezca durante una fase de revelación o de incubación (cf. Moscovici, 1985), lo que podría ser el caso de los sujetos alejados, y, por el contrario, adquiriría la forma de una conversión más directa o explícita durante una fase en la que la contra-norma ya ha sido interiorizada, caso más probable de los sujetos cercanos. Es precisamente la complejidad de estos procesos, y su naturaleza claramente temporal, lo que ha hecho del estudio de la influencia diferida un instrumento metodológico de gran importancia para abordar teóricamente tal orden de fenómenos.

\section{Referencias}

Aebischer, V., Hewstone, M. \& Henderson. M. (1984). Minority influence and musical preference: innovation by conversion not coercion. European Journal of Social Psychology, 14, 23-33. DOISE, W. (1987). Psicología social y relaciones entre grupos. Barcelona: Rol.

KaISER, C. \& MUGNY. G. (1987). Consistance et significations du conflit. In S. Moscovici \& G. Mugny (Eds.), Psychologie de la conversion. Cousset: Delval.

Manss. A. (1987). Minorités et processus de conversion. In S. Moscovici \& G. Mugny (Eds.), Psychologie de la conversion. Cousset: Delval. 
MARTIN, R. (1987). Influence minoritaire et relations entre groupes. In S. Moscovici \& G. Mugny (Eds.), Psychologie de la conversion. Cousset: Delval.

MoscovicI. S. (1981). Psicología de las minorias activas. Madrid: Morata.

MoscovicI, S. (1980). Toward a theory of conversion behavior. In L. Berkowitz (Ed.), Advances in experimental social psychology (Vol. 13). New York: Academic Press.

MoscovicI. S. (1985). Innovation and minority influence. In S. Moscovici, G. Mugny \& E. Van avermaet (Eds.), Perspectives on minority influence. Cambridge: Cambridge University Press.

Moscovici, S. \& MuGNY, G. (Eds.) (1987). Psychologie de la conversion. Cousset: Delval.

Moscovici, S., MUGNY, G. \& P.APASTAMOU, S. (1981). Sleeper effect et/ou effett minoritaire? Etude théorique et expérimentale de l'influence sociale à retardement. Cahiers de Psychologie Cognitive, 1, 199-221.

Moscovia, S. \& PERSONNaz, B. (1980). Studies in social influence V: minority influence and conversion behavior in a perceptual task. Joumal of Experimental Social Psychology, 16, 270-282.

MUGNY, G. (1981). El poder de las minorías. Barcelona: Rol.

Mugny, G., IbÁNez, T., Elejabarreta, F., Iñguez, L. \& Pérez, J.A. (1986). Conflictó, identificación y poder en la influencia minoritaria. Revista de Psicología Social, 1, 39-56.

Mugny, G., Kaiser, C. \& PAPASTAMou, S. (1983). Influence sociale, identification et relations entre groupes: étude expérimentale autour d'une votation. Cahiers de Psychologie Sociale, 19, 1-30.

MUGNY, G. \& PAPASTAMOU, S. (1982). Minority influence and psycho-social identity. European Journal of Social Psychology, 12, 379-394.

MUGNY, G. \& PÉREZ, J.A. (1985). Influence sociale, conflit et identification: étude expérimentale autour d'une persuasion emanquées lors d'une votation. Cahiers de Psychologie Sociale, 6, 1-13.

MUGNY, G. \& PÉREZ, J.A. (1986). Le déni et la raison. Psychologie de l'impact sócial des minorités. Cousset: Delval.

MUGNY, G. \& PÉREZ, J.A. (1987). Le constructivisme social en psychologie sociales: le cas de l'influence des minorités In J.L. Beauvois, R.V. Joule \& J.M. Monteil (Eds.), Perspectives cognitives et conduites sociales. Cousset: Delval.

Mugny, G., PÉrez, J.A., Kaiser, C. \& PAPAStAmou, S. (1984). Influence minoritaire et relations entre groupes: l'importance du contenu du message et des styles de comportement. Revue Suisse de Psychologie, 43, 331-351.

MUGNY, G., RIIUET, D. \& PAPASTAMOU, S. (1981). Influence minoritaire et identification sociale dans des contextes d'originalité et de déviance. Revue Suisse de Psychologie, 40, 314-332.

NeMETH. C. (1986). Differential contributions of majority and minority influence. Psychological Review, 93, 23-32.

PAICHEIER, G. (1977). Norms and attitude change II: the phenomenon of bipolarization. European Joumal of Social Psychology, 7, 4-14.

PEREZ, J.A. \& MUGNY, G. (1985). Influencia minoritaria sobre las opiniones frente al aborto y los anticonceptivos. Estudios de Psicología, 23/24, 29-54.

PEREZ. J.A. \& MUGNY, G. (1987). Paradoxical effects of categorization in minority influence: when being an out-group is an advantage. European Joumal of Social Psychology, 17, 157-169.

Perez, J.A., MugnY, G. \& Moscovici, S. (1986). Les effets paradoxaux du déni dans l'influence sociale. Cahiers de Psychologie Sociale, 32, 1-14.

Personnaz, B. \& GuLlon, M. (1985). Conflict and conversion. In S. Moscovici, G. Mugny \& E. Van Avermaet (Eds.), Perspectives on minority influence. Cambridge: Cambridge University Press.

Personnaz, B. \& Personnaz. M. (1987). Un paradigme pour l'étude de la conversion. In S. Moscovici \& G. Mugny (Eds.), Psychologie de la conversion. Cousset: Delval.

TAJFrI, H. (1982). Social identity and intergroup relations. Cambridge: Cambridge University Press.

TURNER, J.C. (et al.). (1987). Rediscovering the social group. Oxford: Blackwell. 